\title{
Trajectoires éditoriales de la littérature francophone vietnamienne
}

\author{
Pham Van Quang \\ École Supérieure des Sciences sociales et Humaines \\ Université nationale du Vietnam à Ho Chi Minh-Ville
}

\section{Introduction}

Dans le cadre théorique de la sociologie littéraire, ce qui est particulièrement important c'est la procédure de l'analyse des instances de légitimation et de consécration de la production créative des écrivains. Un champ littéraire ${ }^{1}$ n'est institutionnalisé que lorsqu'il y a des acteurs suffisamment institués. Nous parlons ainsi des instances de circulation de la production littéraire. Il s'agit d'abord d'éditeurs ou de maisons d'édition qui sont considérés à la fois comme les espaces de reproduction et de diffusion et comme les représentations de stratégies des publicités littéraires. En effet, dans l'ensemble des milieux socialement marqués par rapport à la vie littéraire ${ }^{2}$ (espaces de presse, académies, sociétés littéraires, attributions des prix littéraires), les éditeurs et les maisons d'édition font partie des acteurs indispensables de la vie littéraire. Pour être reconnues du public professionnel et non professionnel, les oeuvres de création doivent être soumises à un cycle assez complexe où les éditeurs ou maisons d'édition jouent un rôle clé. Quels sont donc l'état des lieux et la fonction des activités de ces acteurs par rapport à la littérature vietnamienne francophone? La réponse est loin d'être précise et est d'autant plus difficile qu'on cherche à l'heure actuelle à mettre en évidence la situation des productions littéraires vietnamiennes dans l'espace francophone. Aussi ne devrons-nous nullement nous étonner de ne trouver aucune observation sur l'édition francophone au Vietnam dans l'étude récente et importante de Luc Pinhas sur Éditer dans l'espace francophone (2005), alors que le Vietnam fait partie officiellement de quarante-neuf États et gouvernements membres de la Francophonie. En effet, à la recherche de leur légitimation, les oeuvres littéraires vietnamiennes ont révélé, au même titre que la situation de leurs auteurs, leurs limites ambiguës et leurs frontières incertaines. Il est en fait malaisé d'établir une histoire du système éditorial au Vietnam. Cela consiste largement à observer des mouvements historiques et politiques qui agissent fortement sur l'espace de production et de commercialisation du livre francophone. Pour déterminer la réalité de la question, nous tentons d'examiner brièvement d'abord la situation éditoriale francophone pendant la période coloniale et ensuite celle qui s'étend de l'indépendance jusqu'à nos jours.

\section{L'ère des imprimeries en Indochine}

Pour la période du début du $\mathrm{XX}^{\mathrm{e}}$ siècle à la fin de la colonisation française, la vie

\footnotetext{
${ }^{1}$ Nous prenons comme point de départ la notion de champ littéraire de Pierre Bourdieu (1992).

2 L'évolution de la vie littéraire vietnamienne se base sur un certain nombre de réseaux de relations entre les auteurs mêmes. Il s'agit d'une façon efficace d'analyser le degré de visibilité. Aussi, avec d'autres moyens de reconnaissance apportés par la procédure à l'analyse des éléments paratextuels, convient-il de développer dans une autre étude le rôle du discours préfaciel qui, pour la plupart des écrivains vietnamiens, est un lieu de complicité leur permettant d'acquérir des prises de position.
}

This work is licensed under a Creative Commons Attribution 3.0 License 
intellectuelle des Vietnamiens est la plupart du temps soumise à l'idéologie et à la politique de l'autorité coloniale. Aussi les structures de la circulation des ouvrages appartiennent-elles au contrôle du gouvernement français de l'époque. Cela dit, le pouvoir censorial est une action importante du gouvernement général de l'Indochine, surtout depuis l'apparition de l'imprimerie. Cette action est bien systématisée et modernisée par Albert Sarraut vers les années 1920 par la mise en oeuvre d'une Direction des Archives et Bibliothèques. Ce qui est synonyme d'une obligation de dépôt légal, appliquée pour les publications imprimées à partir de 1922. Pour cela, l'imprimeur est chargé de déposer deux exemplaires aux bureaux du gouvernement local ou de la mairie. L'un de ces deux exemplaires est remis dans le fonds de la Bibliothèque Centrale de Hanoi, l'autre est envoyé à Paris. Ce régime de la censure est maintenu jusqu'au début de l'année 1945. Ainsi durant plus d'une vingtaine d'années, les imprimeurs et les auteurs sont soumis à l'attribution des permissions d'imprimer. Il n'y avait pas de privilèges pour les productions créatives car elles étaient considérées comme préjudiciables à l'autorité coloniale. Ce pouvoir politique agissait fortement dans la vie intellectuelle des Vietnamiens. Il façonnait la pensée des écrivains. Cette situation difficile peut être illustrée par le cas de Nguyen An Ninh en ces termes:

«Mais le Gouvernement colonial trouve mille moyens pour combattre les journaux annamites de langue française qui ne le flagornent pas. Par exemple, interdiction à tous les imprimeurs d'imprimer ces journaux, interdiction aux fonctionnaires de les lire. Et les fonctionnaires annamites forment une clientèle qu'on ne peut négliger. Des Annamites qui lisent le français, les neuf dixièmes sont fonctionnaires. Quant à ceux qui lisent le français et qui sont commerçants ou propriétaires fonciers, les administrateurs, chefs de province, les convoquent à leur bureau et les engagent à s'abstenir de certains journaux. «Je crois que la sagesse vous conseille...qu'il est de votre intérêt de ne pas lire ces journaux... » Le Gouvernement colonial viole même les correspondances adressées à ces journaux, pour connaitre les noms de ceux qui leur procurent des abonnements » $(1925: 266)$

Il est clair que le champ culturel de l'Indochine de l'époque coloniale est dominé par la politique et l'idéologie du colonialisme qui entrave plus ou moins le développement des activités de publication et de diffusion. D'ailleurs, s'il existe une promotion du développement culturel en Indochine, c'est bien pour servir d'un «tremplin politique ». Or, on se rend très bien compte que la plupart des oeuvres en français écrites par des auteurs vietnamiens visent à la lutte pour l'indépendance du pays. À ce propos, la considération de Charton est judicieuse:

«Toute une littérature de langue française et écrite par les Indochinois existe et publie (sic) des œuvres importantes. Ce n'est pas une littérature officielle ou subventionnée, ou une littérature scolaire et technique, c'est une littérature personnelle, romanesque et, le plus souvent, d'ailleurs, une littérature d'opposition politique » $(1947: 67)$

Dans de telles perspectives politiques, malgré la mise en oeuvre du système réformé de l'enseignement, la structure de l'édition au Vietnam n'a pas semblé être évidente pour ne pas dire qu'il n'existait pas de grandes maisons d'édition. On trouve en effet plutôt des imprimeries qui sont soutenues soit par le gouvernement français, soit par des groupes d'intellectuels vietnamiens. Parmi les imprimeries du premier groupe figurent par exemple l'Imprimerie Impériale, l'Imprimerie du Gouvernement, l'Imprimerie Guillaud et Martinon, l'Imprimerie Rey et Curiol, pour ne citer que les plus connues. Voici un extrait qui exprime clairement le dessein des Français d'implanter l'imprimerie en Cochinchine:

«Un service typographique a été également organisé le 1er février 1862, et a pris le titre d'imprimerie impériale de Saigon. Il comprenait à cette époque 5 ouvriers $(1$ compositeur,

This work is licensed under a Creative Commons Attribution 3.0 License 
imprimeur, 1 écrivain autographe, 1 imprimeur lithographe et enfin 1 relieur) ; le matériel comprenait 2 presses typographiques à bras et 1 presse lithographique. Huit jours après, c'està-dire le 8 février 1862, l'imprimerie était en pleine activité, et la première impression sortie des presses fut une proclamation adressée à l'armée de terre et à la marine par le commandant en chef du corps expéditionnaire, M. le contre-amiral Bonard, qui venait de terminer une courte, mais brillante campagne contre les Cochinchinois opposés à notre domination. D'un autre côté, M. Aubaret, lieutenant de vaisseau et directeur des affaires indigènes, fit venir de Canton trois artistes chinois chargés de graver et d'imprimer en langue du pays un journal officiel destiné à faire comprendre à ces peuples les bienfaits de notre occupation ; ce journal était rédigé en entier par M. Aubaret, aujourd'hui capitaine de frégate et consul de France à Bangkok (Siam), qui connait on ne peut mieux les signes idéographiques usités dans ces contrées lointaines. Aujourd'hui l'imprimerie de la Cochinchine est en pleine prospérité ; le matériel a été augmenté considérablement ainsi que le personnel. Dans l'origine, le journal qui se publiait avait pour titre : Bulletin officiel de l'expédition de la Cochinchine, et paraissait sous format in- $8^{\circ}$, c'est-à-dire qu'il faisait volume ; le nouveau gouverneur, M. de La Grandière, a transformé ce journal, et aujourd'hui il est intitulé Journal officiel de Saigon, et contient des annonces commerciales, les entrées et sorties du port de Saigon, etc., enfin des articles divers sur le pays et les actes officiel. » (L'Empire d'Annam, Grand Dictionnaire Universel du XIXe Siècle de Pierre Larousses)

Sans doute, avec les imprimeries de ce type, les auteurs vietnamiens ne sauraient-ils pas s'attendre à une garantie pour publier leurs oeuvres, surtout celles qui ne se situent pas dans l'intention du gouvernement colonial.

Cependant, certaines de ces imprimeries ont contribué fortement à la fois aux champs culturel et économique en Indochine de l'époque. En effet, on ne peut nier le rôle important des frères Schneider dans l'histoire de l'édition au Vietnam pendant la période de la fin du $\mathrm{XIX}^{\mathrm{e}}$ siècle au début du $\mathrm{XX}^{\mathrm{e}}$ siècle. Ernest Hippolyte Schneider, frère aîné, est libraire et papetier. Il crée une bibliothèque en offrant beaucoup d'ouvrages en prêt. Quant à François-Henri Schneider, considéré comme un célèbre imprimeur, il devient en 1883 le chef d'atelier de l'imprimerie du protectorat. Sa contribution importante à l'histoire de l'édition au Vietnam est marquée surtout vers les années 1885, moment où il prône une imprimerie indépendante. Pour ce faire, il commence par l'impression de cartes postales ainsi que d'ouvrages pour les particuliers. Ce qui est à remarquer en plus c'est qu'il a tenté de moderniser l'imprimerie en faisant venir de France des machines spéciales et performantes. Soutenu particulièrement par le gouverneur général Albert Sarraut, François-Henri Schneider établit en même temps de bonnes relations avec les autorités vietnamiennes locales, ce qui permet de faire de lui un éditeur réputé, d'une part, et d'engager plus ou moins la liberté d'imprimer des ouvrages littéraires en vietnamien comme en français, d'autre part. Le succès de cette imprimerie est donc justifié par la présence des auteurs des premières générations comme Tran Trong Kim, en langue vietnamienne, et des auteurs de langue française, comme Nguyen Trong Hiep (dit Kim Giang) et Le Van Phat : le premier est un poète qui, par ses trente-six quatrains sous le titre Paris, capitale de la France, traduit ses belles impressions par rapport à la capitale de la France où il a passé son séjour ; l'autre témoigne de son admiration pour la tradition annamite en l'illustrant par son recueil de contes et de légendes.

L'apparition de l'Imprimerie d'Extrême-Orient (I.D.E.O.) à Hanoï a aussi marqué un succès de l'autorité coloniale en Indochine. Fondée en 1907, elle se situe au 28 rue Paul Bert (actuellement 24, rue Trang Tien, Q. Hoan Kiem). Considérée comme 
la plus importante d'Indochine, elle est destinée à produire et reproduire des documents officiels du gouvernement. Mais cette imprimerie faisait aussi une place primordiale au domaine des livres de culture et de civilisation vietnamienne. On y voit un certain nombre d'écrits de Pham Quynh et Nguyen Van Huyen. Ces auteurs occidentalisés témoignent aussitôt de leur voix et de leur rôle dans la promotion des valeurs traditionnelles annamites. En même temps, vers les années 1940 apparait la première femme de plume vietnamienne de langue française Trinh Thuc Oanh. Francophile, contestée par ses contemporains vietnamiens, cette femme de lettres, auteur de deux romans et d'un recueil de contes, a cherché, malgré tout, à former avec succès son image par cette imprimerie en tant qu'instance solidement instituée.

À ces imprimeries plus ou moins importantes de l'histoire de l'édition vietnamienne s'en ajoutent d'autres qui sont moins grandes et parfois se trouvent dans une situation financière difficile. À Hanoï, on peut en compter environ une quinzaine qui ont contribué à découvrir le talent des auteurs vietnamiens de langue française, telles l'Imprimerie G. Taupin où les créations littéraires de Pham Duy Khiem sont mises en valeurs; l'Imprimerie Tonkinoise a, elle aussi, accueilli en 1921 Nguyen Phan Long avec Le roman de mademoiselle Lys, considéré comme premier texte romanesque de la littérature vietnamienne francophone. L'Imprimerie Thuy $\mathrm{Ky}$ tente aussi de convaincre le public en transformant les œuvres en valeur marchande. En français, Nguyen Manh Tuong y fait paraître une pièce théâtrale en trois actes Le voyage et le sentiment (1943).

En Cochinchine ou plutôt à Saïgon, les imprimeries occupent déjà une place non négligeable, comme nous venons de le voir en citant les plus importantes soutenues par le gouvernement général. L'Imprimerie de l'Union dont Nguyen Van Cua est directeur ou l'Imprimerie de Joseph Nguyen Van Viet, fondée en 1900, ont bien réussi à l'époque dans la publication de tous les genres notamment en langue vietnamienne. Elles participent évidemment à éditer ou à rééditer des travaux de création littéraire en français. C'est le cas du recueil de poésies Mes heures perdues (1913) de Nguyen Van Xiem ou du livre de Contes et légendes du pays d'Annam (2e édit. 1925) de Le Van Phat. Toujours dans le domaine des textes poétiques, l'Imprimerie J. Aspar permet à Pham Van Ky de publier en 1936 Une voix sur la voie son premier recueil de poésies célèbre, considéré comme le premier à avoir introduit au Vietnam le mouvement surréaliste et donc profondément influencé par des poètes français. Pour autant, l'Imprimerie est pour les écrivains la première instance de consécration. Ainsi, au début du $\mathrm{XX}^{\mathrm{e}}$ siècle, la plupart des écrivains vietnamiens francophones ont eu de la chance de se faire connaitre par les imprimeries soutenues par le gouvernement colonial. De même, l'Imprimerie C. Ardin, dit aussi Maison C. Ardin, fondée en 1868 à Saïgon, joue-t-elle un rôle significatif dans la vie éditoriale en général et dans l'institution de la vie culturelle et intellectuelle des Indochinois en particulier. Cette Imprimerie connait en effet sa grande évolution pour devenir plus tard la Société des Imprimeries et Librairies Indochinoises (SILI). En 1932, Nguyen Phan Long s'y fait un nom par son important recueil de contes Cannibales parpersuasion.

Malgré l'absence de grandes maisons d'édition et de celles qui se spécialisent exclusivement dans le domaine littéraire, le système d'imprimeries à l'époque coloniale, quelles qu'elles soient soutenues ou non par le gouvernement général, reste au 
demeurant la part prestigieuse et appréciable de l'édition francophone au Vietnam en ce qu'elles apportent une contribution non négligeable à la vie culturelle et intellectuelle. Elles sont des acteurs indispensables pour assurer la permanence de la vie littéraire tout en prenant en charge de faire briller la pensée de l'élite et de garantir la dynamique de la vie intellectuelle.

\section{La période sombre de l'édition francophone dans le Vietnam postcolonial}

La décolonisation française est évidemment un événement particulièrement important pour la vie politique des Vietnamiens qui ont pu voir enfin leurs efforts de combattre pour l'indépendance du pays récompensés. Les accords de Genève marquent ainsi en 1954 la fin de la guerre d'Indochine entre la France et la République démocratique du Vietnam, mais ils entrainent en même temps une séparation du Vietnam en deux États. Cette coupure douloureuse deviendra, d'ailleurs, plus tard une source d'inspiration intense pour un certain nombre d'écrivains vietnamiens francophones. Pendant les vingt années suivantes, les mouvements politiques sont dominés par la volonté de réunifier les deux «Vietnams ». Si en 1975, le Vietnam connaît sa réunification, celle-ci est pourtant suivie des années de collectivisation forcée. Dans de telles situations historiques, on comprend sans difficulté que la vie culturelle et intellectuelle bascule dans "une ère du soupçon»: elle est aussi bien ambigüe que menacée. Le premier souci des dirigeants vietnamiens à cette époque est plutôt de rattraper le retard au niveau de l'économie et de normaliser les relations extérieures. C'est aussi la raison pour laquelle, malgré l'adhésion du Vietnam aux institutions de la francophonie depuis 1987 en tant que membre à part entière, le soutien au développement du français n'est jamais mis en jeu comme une promotion culturelle et intellectuelle, mais plutôt comme une stratégie des coopérations économique et diplomatique.

Pour plus de précision au sujet de l'espace francophone du Vietnam, on constate assurément que malgré une centaine d'années de colonisation française, le français n'est jamais arrivé à être une langue véhiculaire. La situation était encore pire lorsque cette langue fut éliminée au Nord dès la chute de Dien Bien Phu, et s'est repliée ensuite au Sud depuis 1975. À cela s'ajoute le fait qu'un changement de régime politique s'accompagne le plus souvent d'un changement de sphères de la vie culturelle et intellectuelle. Ainsi, nombreux sont les intellectuels vietnamiens qui étaient alors conscients que l'indépendance du pays mettait en cause la liberté des individus. Sans doute le choix du français dans ces conjonctures est-il pour certains écrivains la meilleure façon pour exprimer leur pensée. Mais se pose d'emblée le problème de la trajectoire éditoriale de ces écrivains. À ce propos, nous parlerons plus loin du cas de Cung Giu Nguyên.

Revenons sur l'état des lieux de l'édition francophone au Vietnam, il convient de se rendre compte que les mouvements socio-historiques ont conduit à une situation précaire de l'édition, surtout en langues étrangères. On remarque ainsi que les Éditions en Langues étrangères sont la seule maison d'édition où ont été publiés quelques ouvrages de création littéraire en français. Comme en URSS ou en Chine, apparaît au Vietnam en 1957 ce type de maison d'édition qui est chargée de la communication extérieure souvent assignée par l'État. Elle publie donc essentiellement des traductions de livres en 
langues étrangères. Depuis 1964, cette maison est dirigée par le docteur Nguyen Khac Vien, qui était en même temps membre du Comité national des Relations étrangères du Vietnam, et ensuite par l'écrivain Huu Ngoc. Ce sont deux éminents francophones vietnamiens. Dans une certaine mesure, cette maison d'Éditions en Langues étrangères joue un rôle plus ou moins important pour avoir présenté aux lecteurs étrangers des ouvrages d'auteurs vietnamiens. Nous pouvons en effet y trouver des livres de récits de mémoire dont les thèmes portent essentiellement sur la révolution et la guerre. Aussi, en promouvant une littérature de propagande, à partir des années 1960, cette maison d'édition s'est engagée à soutenir des ouvrages collectifs et à les diffuser auprès des lecteurs étrangers. Nguyen Xuan Tram y est connu par sa publication en 1961 De la prison au bagne, recueil de témoignages d'un personnage qui a connu dans les prisons du Sud, pendant 38 mois, de la maison centrale d'arrêt de Quang Tri au bagne de Poulo Condor, l'incarcération et la torture sous la dictature de Ngo Dinh Diem. On y trouve encore le parcours de Lê Thi Tai, Vo Thi Thê, Hoang Liêm, Kim Phuong pour leur récit collectif Et le jour se leva... (1961), ou des auteurs de La Révolution au village. Nam Hong, 1945-1975 (1975), Pham Cuong et Nguyen Van Ba. Nguyen Khai y participe lui aussi avec son petit livre Ceux de Côn Co (1966). Notons cependant que ces Éditions semblent avoir du mal de se maintenir jusqu'à présent. Elles ont en effet connu la fin lors de sa transformation vers les années 1990 en un nouveau nom Éditions The Gioi (Le Monde) qui éditent essentiellement des ouvrages de traduction.

Dans tous les cas, on ne trouve guère un système éditorial francophone à proprement dit au Vietnam, aussi bien à l'époque coloniale que postcoloniale. La situation devient encore plus précaire au lendemain de l'indépendance et de la réunification du pays, de sorte que certaines œuvres en français de Pham Quynh, homme de lettres très connu, étaient mises en doute pour pouvoir être traduites en vietnamien. Les lecteurs vietnamiens ont donc dû attendre plus d'un demi-siècle après la mort de l'auteur pour savourer en vietnamien ses Essais 1922-1932, qui réunissent des articles parus dans France-Indocbine et Indocbine républicaine. Aussi le rôle de l'État est-il extrêmement important dans les politiques du livre, puisque dans le contexte $\mathrm{du}$ Vietnam la structure éditoriale semble lourdement dépendante du pouvoir censorial appartenant évidemment à l'État. En effet, la persistance de la censure politique était particulièrement forte à partir des années 1954, ce qui ne laisse pas de possibilité aux éditeurs d'accueillir des auteurs de livres «problématiques », d'autant plus que les livres en français sont parfois préjugés par le régime comme « nuisibles » à l'État.

\section{Exemple du parcours éditorial de Cung Giu Nguyên}

Cung Giu Nguyên témoigne ainsi d'une trajectoire éditoriale singulière. Auteur de trois romans et de nombreux récits et nouvelles francophones, Cung Giu Nguyên a montré dès les débuts de sa carrière son succès de faire reconnaître ses ouvrages par les éditeurs à l'étranger. Il s'agit par exemple de ses deux premiers romans Le Fils de la baleine (1956) et Le Domaine mandit (1961). Le premier, publié pour la première fois par la Librairie Arthème Fayard où il reçoit un certain succès critique et public. Ce texte est ensuite publié en 1978 chez Naaman de Sherbrooke (Canada). Accepté par ces grandes maisons d'éditions, Cung Giu Nguyên a ainsi largement bénéficié de l'appui des médias les plus influents. Le roman a eu depuis lors un retentissement auprès du public étranger dû à la haute qualité de son contenu et de son style. Le succès de Cung Giu 
Nguyên par la publication de son premier roman lui a permis d'affirmer son entrée littéraire, ce qui amène à former de plus en plus solidement la suprématie de l'auteur, notamment lors de son édition du deuxième roman, toujours chez Fayard.

Au contraire, Le Boujoum, troisième roman de Cung Giu Nguyên, n'a pas eu cette chance éditoriale. En effet, ce chef-d'œuvre est apparu dans un contexte fâcheux. D'abord, son manuscrit prend forme en 1980, après quatre années de gestation, plus précisément depuis la réunification du Vietnam en 1975. Mais Le Boujoum n'a pas reçu de soutien des éditeurs étrangers pour diverses raisons. La première personne à qui l'auteur avait recours en 1980 pour transcrire le manuscrit en version dactylographique a abandonné le travail après la lecture de quelques dizaines de pages. La raison est sans doute due au style romanesque difficile recherché par l'auteur'. Après cet échec dans la légitimation, c'est l'auteur même qui a dactylographié son manuscrit dans l'espoir de pouvoir être traduit en vietnamien comme son premier roman Le Fils de la baleine. Malheureusement, personne n'a pu répondre à l'attente de l'auteur du Domaine mandit. Cung Giu Nguyên a alors fait lui-même la traduction de son œuvre. En version vietnamienne sous le titre Thái Huyền ${ }^{4}$, la première partie du roman a été éditée en 1994 par Dai Nam, une petite maison d'édition vietnamienne en Californie - États-Unis. Cependant, la situation difficile du marché du livre n'a pas permis à la seconde partie du roman de voir le jour. Aussi peut-on constater que le premier obstacle - et cela est bien clair - qui empêche la parution du Boujoum s'explique par la lacune d'un système éditorial francophone au Vietnam.

À cet empêchement qui vient de l'extérieur s'ajoute apparemment la question du type d'écriture choisi par l'auteur qui exige en effet du lecteur un énorme engagement pour la construction de sens du texte. En ce sens, le lecteur se désigne comme :

« [Celui qui] devenait le lieu où l'unité du texte se produisait, dans la destination au lieu de son origine, mais ce lecteur n'était pas plus personnel que l'auteur tout juste déboulonné, et il s'identifiait lui aussi à une fonction : il était ce quelqu'un qui tient rassemblées dans un même champ toutes les traces dont est constitué l'écrit » (Mollier et al. 2002 :77).

Cette restriction par rapport au lecteur potentiel traduit en partie l'insuccès du roman d'autant plus que se manifeste une situation précaire des lecteurs francophones vietnamiens.

Le sort du livre de Cung Giu Nguyên est donc largement tributaire des possibilités de la commercialisation. Pour autant, l'auteur ne gagne pas sa place. Cependant, on peut mesurer le succès d'un livre en se basant non sur l'intérêt économique mais sur le symbolique. Dans une telle sphère, Cung Giu Nguyên est sans doute pris dans sa vision que tout se passe comme s'il n'y avait rien d'autre en dehors de sa conscience. L'auteur est d'ailleurs convaincu que la vie de son œuvre durera plus longtemps que celle des régimes qui cherchent à la détruire. C'est pour cela que bien qu'on doive attendre plus de vingt ans après la formation de son manuscrit pour voir

\footnotetext{
3 Voir « Préface » de Thái Huyền.

${ }_{4}$ Notons par ailleurs que l'ambiguïté de l'écriture romanesque se manifeste dès le titre du livre, en français comme en vietnamien : si le terme «Boujoum» n'existe pas dans le dictionnaire français, il en va de même avec le terme «Thái Huyền » en vietnamien. Le lecteur soucieux de comprendre l'intention de l'auteur serait donc amené à lire la préface de Thái Huyền.
} 
naitre ce roman en version française complète - il est publié en 2002 par le Cunggiunguyen Center, fondé au Texas par des élèves de Cung Giu Nguyên en vue de collecter et maintenir des documents de cet auteur ainsi que de promouvoir ses productions littéraires. Le Boujoum est actuellement considéré comme le vrai chefd'œuvre de Cung Giu Nguyên. C'est en fait un grand roman philosophique de 654 pages. Il est clair que le public visé par l'écrivain est un public restreint. Aussi le succès ou l'échec pour cet écrivain ne tient pas nécessairement à observer si son ouvrage atteint un gros tirage. Seul importe pour l'écrivain d'être lu. Sa volonté s'est donc réalisée puisque la Bibliothèque des Sciences Générales de Ho Chi Minh-Ville dispose actuellement d'un exemplaire de ce roman sur ses rayons ${ }^{5}$.

À la lumière de la trajectoire éditoriale de Cung Giu Nguyên, on se rend compte par ailleurs que son parcours littéraire illustre une situation particulière. En effet, l'auteur n'a pas quitté le pays ; il est resté toujours à la recherche de sa consécration au sein du territoire où la production francophone était déjà en sommeil. Est-ce une contradiction de ses textes autant que celle de la vie l'auteur? Il n'y a pas de réponse définitive. Mais une chose est sûre, c'est que Cung Giu Nguyên fait partie des grands auteurs du champ littéraire francophone vietnamien.

\section{Une littérature périphérique d'un champ éditorial central}

Il appartient en effet à des écrivains de légitimer ou de faire reconnaître leurs discours littéraires. Aussi les écrivains vietnamiens francophones prennent-ils conscience des stratégies pour rendre public leurs écrits. Cela amène directement à la procédure éditoriale, ou pour mieux dire à la stratégie éditoriale. On a tout à fait raison de s'inquiéter de l'existence de la littérature vietnamienne francophone ainsi que de la constitution de son champ lorsque son réseau éditorial représente de plus en plus sa fragilité ou son absence totale sur le territoire vietnamien. À part Cung Giu Nguyên qui a traversé tout un siècle au Vietnam mais qui a cherché à imprimer à l'étranger, plus précisément en France, au Canada et aux États-Unis, la plupart des écrivains professionnels vietnamiens s'installent à l'étranger. Il est donc tout à fait logique que leurs ouvrages soient édités et aussi diffusés souvent dans le pays où ils vivent. Ainsi faut-il reconnaître encore une fois que la littérature vietnamienne s'engage dans une mésaventure et dans une contradiction où il n'y a, semble-t-il, pas le choix favorable pour les auteurs. Cette situation se manifeste plus clairement par le fait que matériellement les livres vietnamiens sont pour la plupart publiés à Paris, centre éditorial, mais symboliquement ils constituent un domaine en périphérie. C'est le cas par exemple de Tran Van Tung, Pham Van Ky, et Pham Duy Khiem, ceux qui appartiennent aux premières générations des écrivains vietnamiens dont le pays de résidence est la France. Par leurs parcours éditoriaux en France, ces auteurs devaient être conscients de leur conquête d'un lectorat français ou francophone. Mais d'une façon ou d'une autre, les grandes maisons d'édition au sein de Paris deviennent pour nos écrivains un gage de plus de la légitimation et de la visibilité de leurs œuvres.

La trajectoire de Tran Van Tung se révèle particulièrement variée à la fois dans ses écrits et dans son projet éditorial. Pour ce dernier, il passe des petites aux grandes

5 http://gslhcm.org.vn/libol/search/index.asp.

This work is licensed under a Creative Commons Attribution 3.0 License 
maisons. Les éditions J. Susse, lorsqu'elles tentent de s'approprier des domaines différents qui vont «des sciences humaines aux voyages", permettent à notre écrivain d'éditer ou rééditer ses genres divers: Bach-Yên ou la Fille au coeur fidèle (roman: 1946), Rêves d'un campagnard annamite (roman autobiographique: réédité en 1946), Chants du Dragon d'or (recueil de poèmes: 1945), L'Annam: pays du rêve et de la poésie (recueil de poèmes: 1945). Vers les années 1940, Mercure de France publie ses romans et ses recueils de poèmes et de contes: Rêves d'un campagnard annamite (roman: 1940), Muses de Paris (recueil de poèmes: 1942), Le Coeur de diamant (contes d'Annam: 1944). Nous pourrions pousser plus loin l'énumération, en mentionnant Grasset, Belle-page, Parc et Serg.

Du côté de Pham Van Ky, on observe son parcours éditorial remarquable. Gallimard et Seuil sont les plus importantes maisons d'édition qui font valoir sa trajectoire en particulier et la visibilité de la littérature vietnamienne en général. En effet, avec ces grandes maisons d'édition, les œuvres littéraires de Pham Van Ky constituent un domaine de très grande tenue. Ou pour mieux dire, les ouvrages de création de cet auteur portent si haut le flambeau en rayonnant de mille feux au sein de ces importantes maisons d'édition. Voyons les «avis aux auteurs» de Gallimard pour comprendre que les livres de Pham Van Ky ne déshonorent pas le catalogue de la littérature mondiale:

«Par la quantité et la qualité des auteurs qu'il publie, Gallimard possède le catalogue de la littérature le plus prestigieux de l'édition française et peut-être même de la littérature mondiale. Il cherche dans les jeunes auteurs ceux qui sont 'porteurs d'une oeuvre'. Il écarte impitoyablement les amateurs et les velléitaires. Les débutants qui publient chez Gallimard sont très rares. Ceux qui y parviennent disposent d'un sérieux atout pour leur avenir en littérature, même s'ils n'y font qu'un court passage [...] » (Gaillard 2005 :285).

Ainsi, les lecteurs épris de grands ouvrages chez Gallimard ont pu découvrir Pham Van Ky avec quatre textes romanesques qui font de l'auteur un écrivain de valeur. Y sont successivement publiés Les Yeux courroucés (1958), Les Contemporains (1959), Perdre la demeure (1961) et Des Femmes assises çà et là (1964).

Il faut remarquer d'ailleurs que dans le domaine des textes romanesques, Pham Van Ky témoigne de son succès immédiat dès la publication de son premier roman Frères de sang (1947) chez Seuil, un éditeur très sélectif, dont la politique éditoriale est de "publier des oeuvres originales ». Ce premier roman constitue largement la suprématie de l'écrivain francophone vietnamien au milieu de critiques. Ayant traité de façon impressionnante du thème de l'interaction entre deux mondes - l'Orient et Occident -, deux arts que l'auteur doit combiner, ce roman par son trait caractéristique évoque la curiosité du lecteur européen et attire beaucoup l'attention des critiques contemporains.

Le succès éditorial de Pham Van Ky doit être aussi marqué par l'accueil de la maison de Bernard Grasset en 1954. On constate qu'à côté de Gallimard, les éditions Grasset étaient considérées vers les années 1930 comme une maison incontournable pour la littérature française. En effet, avec le roman Celui qui régnera (1954) où l'auteur continue à aborder la crise supportée par un individu qui se trouve entre deux civilisations, Pham Van Ky a bénéficié d'un soutien conjoint de la grande maison d'édition en contribuant à la promotion de la littérature francophone. Pour compléter la liste des éditeurs qui participent à la reconnaissance des livres de création littéraire de 
Pham Van Ky, il nous faut citer encore les Éditions-Ibis, les Éditions Fasquelle qui fusionnent avec Bernard Grasset en 1959.

Les Éditions Plon accordent également une place importante à la littérature francophone tout en publiant des auteurs de prestige et de tradition de langue française. Dans les domaines du roman et du récit autobiographique, cette maison accueille particulièrement le récit d'amour, d'aventure ainsi que les textes à caractère historique et religieux. Pham Duy Khiem se range dans les auteurs privilégiés qu'il s'agisse des personnalités de l'intelligentsia (les politiciens, les ambassadeurs, etc). C'est ainsi qu'entre 1957 et 1958, cet éditeur de littérature de fiction parvient à porter vers le public deux fameux romans autobiographiques de cet ancien normalien et ambassadeur vietnamien. Le premier Nam et Sylvie (1957) se situe exactement dans le type phare de roman d'amour, où s'esquisse le sujet d'une liaison poétique entre un Vietnamien et une Française. Mais c'est une liaison impossible qui ne voit pas de lendemain. Le second La Place d'un homme (1958) retrace l'histoire d'un intellectuel annamite qui pour l'honneur de la patrie a quitté son poste de professeur pour s'engager en 1939 dans l'armée française. En cela, l'auteur veut justifier et affirmer la valeur et la position d'un Annamite au regard des Français. Par son service dans l'armée française, Pham Duy Khiem tente d'effacer les malentendus dans la relation entre les Annamites colonisés et les Français colonisateurs.

Il est donc incontestable que certains grands écrivains vietnamiens d'expression française sont largement lus, surtout par le lectorat de la métropole, grâce à une grande partie de leur parcours éditorial. Alors que la situation actuelle de l'édition littéraire francophone est presque absente au Vietnam et que la littérature vietnamienne est fortement dépendante des systèmes éditoriaux étrangers, les écrivains de jeune génération cherchent toujours à se faire reconnaitre en France et dans certains autres pays francophones comme le Canada ou la Belgique. Leur trajectoire éditoriale semble être plus avantageuse à la faveur des mutations géographiques mondiales ainsi que du champ culturel essentiellement de la linguistique.

On trouve ainsi que les écrivains sont aujourd'hui chaleureusement accueillis dans les plus grandes maisons d'édition françaises, depuis les plus classiques jusqu'aux plus récentes, comme Stock, Gallimard, Flammarion, Actes Sud, L'Harmattan, la Différence, Picquier, etc. Il en est ainsi pour le cheminement éditorial de deux femmes de lettres Kim Lefèvre et Anna Moï. Ces dernières ont en commun la reconnaissance rapide chez Flammarion, l'une des plus anciennes maisons d'édition françaises, où la même année 2006, nos deux écrivaines publient chacune un roman. La publication de Les Eaux mortes du Mékong de Lefèvre permet au lecteur d'y découvrir «l'histoire d'un premier amour à la douceur tragique », vécue par l'auteur même avec un jeune lieutenant français au moment où elle était réfugiée avec son père dans un village du Sud-Vietnam ${ }^{6}$. Ce roman est immédiatement sélectionné comme un ouvrage de qualité et élu, en 2006, Livre vedette par Le Grand Livre du mois, Club français de l'Actualité littéraire. Il est ensuite réédité, en 2007, par les Éditions Succès du Livre. Quant au roman Violon d'Anna Moï, Chevalier des Arts et des Lettres, on y reconnait un talent confirmé dans le monde des lettres francophones : l'auteur a très habilement manipulé

6 Voir le quatrième de couverture du livre, Flammarion, 2006.

This work is licensed under a Creative Commons Attribution 3.0 License 
le langage en faisant revivre ses souvenirs et son expérience par les époques et par chaque personnage. Cependant, si Anna Moï passe pour la première fois chez Flammarion, elle est déjà une auteure connue chez Gallimard avec ses deux autres romans Riz.noir (2004) et Rapaces (2005) et son bref essai sur la francophonie Espéranto désespéranto (2006).

Après Retour à la saison des pluies publié chez B. Barrault en 1990, Kim Lefèvre cherche en 1994 à se lier avec les Éditions Stock pour la publication de Moi, Marina la Malinche. Cet éditeur de littérature, qui est le plus ancien de France, s'intéresse particulièrement à la fiction étrangère à laquelle appartient l'ouvrage de Kim Lefèvre, qui, par la richesse des connaissances historiques dépeint le portrait sensible d'une femme prise entre deux cultures patriarcales et ethnocentriques à l'époque de la colonisation.

Le cheminement éditorial de ces deux écrivaines est immanquablement mis en évidence par leur place reconnue aux Éditions de l'Aube. Fondées en 1987 et dirigées actuellement par le sociologue Jean Viard, ces éditions ont pour politique de faire une place importante à «des auteurs peu libre de s'exprimer dans leurs pays ». D'où l'exploration des littératures traduites issues de différents domaines parmi lesquels se trouve une partie considérable de la littérature vietnamienne représentée notamment par Nguyen Huy Thiêp. Si le chemin de la «nouvelle» littérature vietnamienne francophone fut sans doute éclairé par le premier récit Métisse blanche (1989) de Kim Lefèvre, en publiant deux recueils de nouvelles L'Écho des rizières (2001) et Parfum de pagode (2003) de Anna Moï, les Éditions de l'Aube continuent de faire découvrir le talent de cette écrivaine en la sélectionnant comme leur auteur-phare littéraire.

Parmi les maisons d'édition modernes qui contribuent à diffuser et à circuler le livre vietnamien, les Éditions Picquier joue un rôle prépondérant. Fondée en 1986, cette maison s'est attachée à publier principalement les littératures d'Extrême-Orient. Avec la certitude que "l'Asie est suffisamment vaste pour qu'on ne s'occupe que d'elle », elle a créé plusieurs collections, ce qui lui permet d'être une maison singulière dans le paysage éditorial français. C'est dans cette politique progressivement ouverte qu'est née la collection «Vietnam » actuellement sous la responsabilité de l'écrivain et traducteur Phan Huy Duong. Cette collection compte à la fois des auteurs vietnamiens traduits et des auteurs francophones. En effet, dans les premières années de ce $\mathrm{XXI}^{\mathrm{e}}$ siècle, la littérature vietnamienne d'expression française dans la métropole connaît son essor grâce au soutien important des Éditions Picquier. On y apprécie particulièrement des oeuvres de création romanesques des deux soeurs Tran Nhut (Thanh Van et Kim). Cette série de romans est publiée sous le titre des Enquêtes menées par le mandarin Tân et ses acolytes, plongeant le lecteur dans une société du XVII ${ }^{\mathrm{e}}$ siècle avec une civilisation presque méconnue d'un Vietnam ancien avec ses moeurs et ses interrogations. Il est à remarquer d'ailleurs qu'avec une telle diffusion régulière de ces deux jeunes écrivaines, la littérature vietnamienne francophone est mise en valeur dans son discours, non seulement parce qu'elle retient l'attention sur les enjeux linguistiques ou sur la promotion du français, mais parce qu'elle ouvre aux lecteurs une nouvelle vision du Vietnam, pays qui subit au fil de l'histoire maints changements idéologiques: du confucianisme au taö̈sme en passant par le colonialisme et le marxisme. 
C'est sans doute dans cette même ligne que plusieurs autres éditions françaises ont comme politique d'éditer de la littérature étrangère. Il s'agit surtout du cas des maisons de moyenne ou petite structure, comme par exemple Christian Bourgois, L'Harmattan ou La Différence. Crée en 1966, la maison Christian Bourgois consacre $80 \%$ de son catalogue à la littérature étrangère. Linda Lê y est venue comme une écrivaine de nouvelles tendances pour lesquelles «la littérature n'est pas faite pour les acquittés, elle n'est pas faite pour les élus. Elle est dans le camp des victimes et des sacrifiés, dans le camp des condamnés qui essayent, comme moi, de trouver leur salut et qui se cassent les dents » (Lê in Christian Bourgeois). Après avoir publié très tôt quelques livres chez La Table ronde et Points, Linda Lê s'attache depuis 1993 aux Éditions Christian Bourgois avec une quinze romans.

Les éditions L'Harmattan aident également à l'institution de la littérature vietnamienne francophone parmi d'autres aires géographiques. Son catalogue de l'Asie dont font partie les sections «Indochine » et «Vietnam» est de plus en plus riche des ouvrages des auteurs vietnamiens, surtout dans les domaines des récits de mémoires ou de témoignages et des recueils de contes. Cette maison regroupe ainsi des auteurs de générations différentes qui sont soit des écrivains occasionnels ou sans carrière, soit des jeunes écrivains amateurs. Pour les premiers, on y retrouve par exemple Dong Sy Hua avec De la Mélanésie au Vietnam: itinéraire d'un colonisé devenu francophile (1993), Le Huu Khoa avec Itinéraire d'un petit mandarin (1997), Minh Tri avec son récit Saigon à l'beure de Hanoi: 1975-1980 (2000) et Huynh Ba Xuan avec Oublié 23 ans dans les goulags Viet-minh 1953-1976 (2003).

Dans le second groupe se rassemblent certains romanciers nouveaux venus : Bach Mai (Hier Saigon: 2009), Tran Thi Hao (Jeune fille et la guerre: 2007) et un grand nombre de conteurs tels Pham-Dao Thérèse, auteur de L'Ogresse et les enfants (2005) et Le cheval d'étain (2006), Do-Lam Chi Lan, docteur en psychologie de l'Université Paris Descartes, avec ses trois ouvrages: La mère et l'enfant dans le Vietnam d'autrefois (1998), Chants et jeux traditionnels de l'enfance au Vietnam (2002) et Contes du Vietnam, enfance et tradition orale (2007). À côté des livres en français proprement dits, L'Harmattan édite aussi pour sa collection «Contes des quatre vents » des recueils de contes bilingues vietnamiens. Mais parmi les livres bilingues, les deux ouvrages de poèmes Parfums (2005) et Déserts (2009) de Nguyen Hoai Huong proposent une écriture prometteuse à double voix ${ }^{7}$ de la jeune poète à travers qui s'enrichit sans doute l'espace de la poésie vietnamienne francophone.

Dans la situation actuelle de l'édition du livre francophone vietnamien, il faut compter les efforts des Éditions de la Différence auxquelles Nguyen Huu Khoa doit jusqu'en 1995 la publication de sa trilogie de romans: La Montagne endormie (1987), Le temple de la félicité (1991) et Métamorphose de la tortue (1995). Ces textes romanesques donnent aux lecteurs à découvrir les valeurs philosophiques ainsi que intellectuelles, mystiques et spirituelles qui s'enracinent dans l'histoire orientale.

On ne saurait pour la présente étude énumérer toutes les maisons d'édition qui tentent de s'approprier une part du marché de l'édition de circulation du livre

7 La poésie de Nguyen Hoai Huong est essentiellement bilingue française et vietnamienne. 
francophone vietnamien, surtout par rapport à de petites maisons. Cela demanderait une recherche plus développée et plus systématique. Pour terminer cette présentation de la situation éditoriale des ouvrages en question, il est nécessaire de citer finalement la librairie Sudestasie. Créée officiellement le 14 octobre 1971 au sein de Paris et dirigée par le Père Nguyen Dinh Thi, cette librairie est d'abord spécialisée dans les documents des pays d'Asie du Sud-Est ${ }^{8}$, en particulier du Vietnam. Cette librairie édite aussi depuis 1980 le Magazine Sudestasie. Consacrée à la littérature vietnamienne, elle aide actuellement à faire connaitre certains auteurs occasionnels comme Thanh Trâm avec sa Légende du ver à soie publiée en 1986. Mais on y retrouve aussi un poète et romancier renommé Pham Quat Xa, lauréat de l'Académie française (poésie 1993). L'auteur y fait publier en 1997 son premier roman Le rêve du papillon, livre sélectionné parmi les oeuvres représentatives de l'UNESCO.

\section{En guise de conclusion}

Nous venons d'examiner la généralité des trajectoires éditoriales de la littérature francophone vietnamienne. Quelles que soient les vicissitudes matérielles que subissent les œuvres dans leur processus de production et de diffusion, les valeurs symboliques que leur apporte l'institution éditoriale sont indéniables. Les imprimeries à l'époque coloniale, bien qu'elles soient dépendantes des idéologies du gouvernement, ont grandement concouru à la vie intellectuelle des Vietnamiens en général et à la transfiguration d'une littérature francophone en particulier. Elles deviennent par ailleurs un élément important dans la formation de la suprématie esthétique et sociale des écrivains, tout en contribuant à la consécration de leurs textes à l'échelle internationale. Il en va de même pour les auteurs contemporains, exilés ou métis pour des raisons sociopolitiques, leur œuvres retrouvent toujours une place particulière dans le système éditorial du pays d'accueil. Cependant, dans la volonté de reconnaittre à l'heure actuelle « une littérature-monde »", une littérature sans frontières, faut-il encore nécessairement parler d'une structure d'édition nationale?

8 Elle a récemment ouvert un rayon Indonésie-Malaisie tout en collaborant avec l'association francoindonésienne pour sa collection du Banian.

${ }_{9}^{9}$ Michel Le Bris et Jean Rouaud (2007) ou Pascale Casanova (2008). 


\section{Bibliographie}

Bourdieu, Pierre. Les règles de l'art. Genèse et structure du champ littéraire. Paris : Seuil, 1992.

Brocheux, Pierre et Daniel Hémery. Indochine : la colonisation ambiguë 1858-1954. Paris : La Découverte, 1995.

Casanova, Pascale. La République mondiale des lettres. Paris: Seuil, coll. «Points », 2008.

Charton, Albert. «L'évolution culturelle de l'Indochine. » Politique étrangère 1, 12e année, (1947) : 51-68.

Gaillard, Roger, Audace. Annuaire à l'Usage des Auteurs cherchant un éditeur. Vitry : L'Oie plate, 2005.

La guerre "française » d'Indochine (1945-1954). Les sources de connaissance.

Bibliographie, filmographie, documents divers. Dir. Ruscio Alain. Paris: Les Indes Savantes, 2002.

Linda Lê. «Fiche auteur. » Christian Bourgois éditeur. <http://www.christianbourgoisediteur.com/fiche-auteur.php?Id=8>. Consulté le 14 avril 2010.

Lefèvre, Kim. Les Eaux mortes du Mékong. Paris : Flammarion, 2006.

Mollier, Jean-Yves et al.. Où va le livre? Dir. Jean-Yves Mollier. Paris : La Dispute, 2002.

Montagnon, Pierre. France-Indochine. Un siècle de vie commune (1858-1954). Paris : Éditions Flammarion, département Pygmalion, 2004.

Nguyen, An Ninh. «La France et l'Indochine.» Europe, revue mensuelle 31 (1925): 257-277.

Pinhas, Luc. Éditer dans l'espace francophone. Paris : Alliance, 2005.

Michel Le Bris et Jean Rouaud (Éd.). Pour une littérature-monde. Paris : Gallimard, 2007.

Trinh, Van Thao. L'école française en Indochine. Paris : Karthala, 1995.

Viatte, Auguste. Histoire comparée des littératures francophones. Paris : Nathan, 1980. 